\title{
Pharmacological Treatments that Facilitate Extinction of Fear: Relevance to Psychotherapy
}

\author{
Michael Davis, Karyn M. Myers, Jasmeer Chhatwal, and Kerry J. Ressler \\ Emory University School of Medicine, Center for Behavioral Neuroscience, and the Yerkes National Primate Center, \\ Atlanta, Georgia 30329
}

\begin{abstract}
Summary: A great deal is now known about the mechanisms of conditioned fear acquisition and expression. More recently, the mechanisms of inhibition of conditioned fear have become the subject of intensive study. The major model system for the study of fear inhibition in the laboratory is extinction, in which a previously fear conditioned organism is exposed repeatedly to the fear-eliciting cue in the absence of any aversive event and the fear conditioned response declines. It is well established that extinction is a form of new learning as opposed to forgetting or "unlearning" of conditioned fear, and it is hypothesized that extinction develops when sensory pathways conveying sensory information to the amygdala come to engage GABAergic interneurons through forms of experience-dependent plas-
\end{abstract}

ticity such as long-term potentiation. Several laboratories currently are investigating methods of facilitating fear extinction in animals with the hope that such treatments might ultimately prove to be useful in facilitating exposure-based therapy for anxiety disorders in clinical populations. This review discusses the advances that have been made in this field and presents the findings of the first major clinical study to examine the therapeutic utility of a drug that facilitates extinction in animals. It is concluded that extinction is an excellent model system for the study of fear inhibition and an indispensable tool for the screening of putative pharmacotherapies for clinical use. Key Words: Extinction, fear, NMDA, D-cycloserine, psychotherapy, cognitive behavioral therapy.

\section{INTRODUCTION}

Anxiety disorders affect roughly $16 \%$ of the U.S. population. Although several medications are effective in treating anxiety disorders, they typically are only palliative. Methods to treat, as opposed to simply blunt, pathological levels of fear and anxiety are sorely needed. Cognitive behavioral therapy (CBT) has proven to be quite effective in treating a number of anxiety disorders. In particular, exposure-based CBT is extremely effective in treating phobic disorders and is regarded widely as the treatment of choice for specific phobias. Exposure-based CBT depends on the process of fear extinction, which can be studied objectively in animals. Several laboratories are studying the cellular processes involved in fear extinction with an eye to developing pharmacotherapies for use as adjuncts to exposure-based CBT for more complex anxiety disorders such as panic, post-traumatic stress, and obsessive-compulsive disorders, as well as

Address correspondence and reprint requests to Michael Davis, Emory University, Department of Psychiatry, Yerkes National Primate Center, 954 Gatewood Drive Northeast, Atlanta, GA 30329. E-mail: mdavis4@emory.edu. social phobia. This paper will review pertinent aspects of animal models of conditioned fear and extinction of fear with a focus on experiments that have developed pharmacological methods to facilitate extinction of fear in rodents. We will conclude by describing the first attempt to translate one of these basic animal studies into a human clinical trial, in which exposure-based CBT was improved successfully by coadministration of a drug called D-cycloserine.

\section{Acquisition of conditioned fear}

A great deal is now known about the circuitry and cellular events underlying conditioned fear acquisition and expression. ${ }^{1}$ In the laboratory, these processes are examined through the study of Pavlovian fear conditioning, a form of simple learning in which an organism (typically a rat, mouse, monkey, or human) is presented with an aversive event (unconditioned stimulus or US) such as a shock or blast of air shortly after the onset of a cue (conditioned stimulus or CS) such as a light or tone. As a function of the contingent pairings of these stimuli, the cue (which elicited little or no reaction before conditioning) comes to produce a constellation of autonomic and behavioral reactions that operationally define 
a state of conditioned fear. Increases in blood pressure and respiration, sweating, and pupillary dilation occur. Rodents tend to hold very still (freezing), and all species show increased startle reflexes elicited by loud noises in the presence of the cue (called "fear-potentiated startle").

Converging evidence from many laboratories indicates that a brain structure called the amygdala, located in the temporal lobe, is critically involved in both the formation and expression of aversive memories. ${ }^{2}$ The amygdala receives highly processed information from all sensory modalities and projects widely to parts of the brain involved in the autonomic and somatic aspects of fear and anxiety. When the amygdala is damaged or inactivated in animals, the acquisition and expression of conditioned fear are blocked. When humans look at pictures of people who are afraid, remember traumatic events, or perceive cues previously paired with shock, there is an increase in blood flow to the amygdala, as assessed with imaging techniques such as positron emission tomography or functional magnetic resonance imaging. Fear learning appears to involve movement of calcium into amygdala neurons followed by a complex pattern of intracellular changes that presumably leads to long-term structural changes, allowing conditioned fear to become more or less permanent.

A major problem in post-traumatic stress disorder (PTSD) and certain other types of anxiety disorders is an inability to suppress or inhibit terrible memories. These patients often do not respond to safety signals. For example, patients suffering from PTSD seem not to benefit from their presence of other people, such as their spouse, that help those without the disorder cope with painful fear memories. ${ }^{3}$ Hence, an important area of inquiry concerns the ways in which unwanted memories are inhibited and the reasons that they fail to be inhibited following traumatic fear conditioning.

\section{Inhibition of conditioned fear}

Behavioral techniques to inhibit or suppress conditioned fear in the laboratory have been known for some time. Among the simplest of these is extinction, in which a subject that previously was trained to fear a cue through pairings of the cue with an aversive event subsequently is exposed to the cue in the absence of the aversive event and the fear conditioned response declines. This protocol will be familiar to clinicians specializing in the treatment of fear disorders in humans because many of the most commonly employed therapeutic techniques such as flooding, systematic desensitization, imaginal therapy, and virtual reality-based therapies are essentially extinction procedures involving exposure to the feared object or situation in the absence of any overt danger. Extinction of fear is thus an excellent model system for the study of fear inhibition and one whose implications for applied work are particularly straightforward. Behavioral studies of extinction have been ongoing since the late 19 th century, and biological research conducted over the last decade has revealed some of the basic mechanisms of extinction and is beginning to suggest some practical, readily applicable modifications of and/or adjuncts to existing forms of therapy.

\section{EXTINCTION: BEHAVIORAL FEATURES, THEORIES, AND BASIC NEUROBIOLOGICAL MECHANISMS}

It is important to point out that the term "extinction" is used in several different ways. Extinction may refer to 1) the experimental procedure used to produce a decrement in the fear response; 2) the decremental effect of this procedure upon the fear response; and 3) the theoretical process responsible for that effect. Moreover, extinction can be measured both at the time the fear-inducing cue is presented in the absence of the aversive event and at a later time. We will define the experimental procedure as extinction training, the decrement in the fear response measure during extinction training as within-session extinction, and the decrement measured at some interval after extinction training as extinction retention. The term extinction will be reserved for the theoretical process underlying the loss of the conditioned fear response.

Extinction is known not to result from forgetting because the fear-conditioned response lasts months, even years, in the absence of additional training after fear acquisition $^{4}$ (FIG. 1A). The mechanism of extinction has been the subject of some debate historically. Some theories have described extinction as an "unlearning" process that results when the fear-inducing cue no longer predicts delivery of the aversive event. ${ }^{5}$ Others have emphasized habituation to the cue as a function of repeated presentation of the cue in the absence of the aversive event. ${ }^{6}$ However, three major pieces of evidence challenge these views. First, the expression of extinction dissipates (i.e., the extinguished response reappears) over time after extinction training, a phenomenon known as spontaneous recovery ${ }^{7}$ (FIG. 1B). Second, extinguished responses reappear when the subject is tested outside of the context of extinction training, a phenomenon known as renewal ${ }^{8}$ (FIG. 1C). Third, extinguished responses reappear when the subject is exposed to unsignaled presentations of the aversive event after extinction training, a phenomenon known as reinstatement ${ }^{9}$ (FIG. 1D). In each of these cases, the reappearance of extinguished responses in the absence of additional pairings of the cue and the aversive event indicates that the fear response cannot have been unlearned. Habituation to the cue potentially can account for spontaneous recovery and reinstatement because habituation dissipates over time and is disrupted by stressors, ${ }^{10}$ but habituation cannot easily account for renewal because habituation does not seem 
A. Extinction is not the same as forgetting

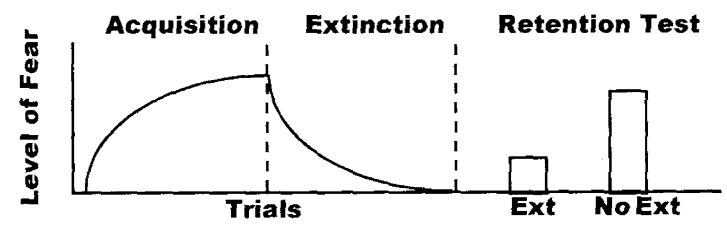

B. Spontaneous recovery - fear return over time

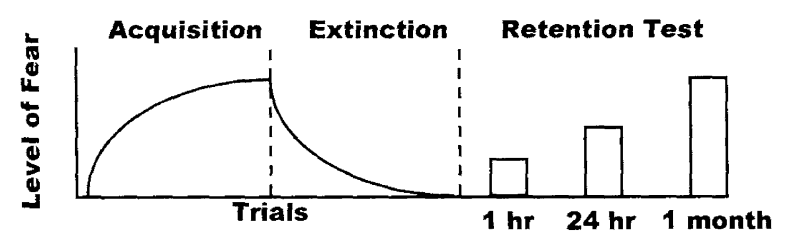

C. Renewal - fear return in non-extinction context

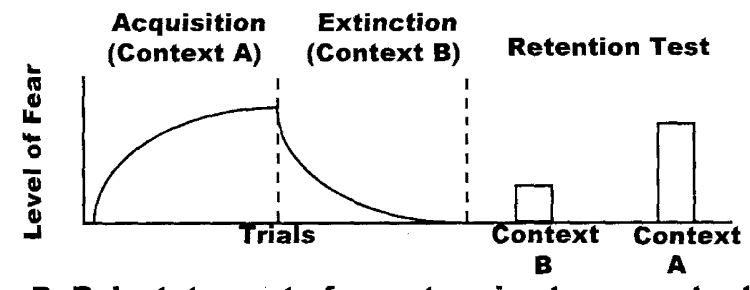

D. Reinstatement - fear return in stress context

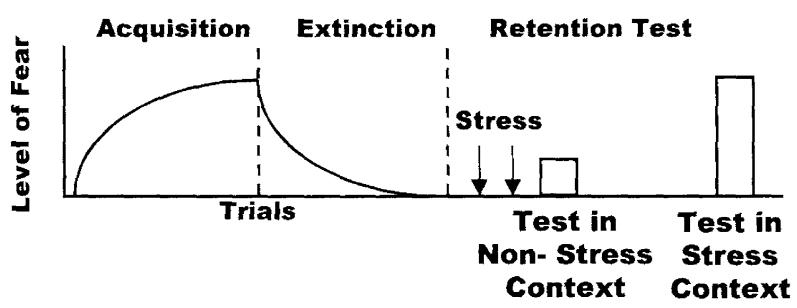

FIG. 1. Behavioral features of extinction. A: Extinction is not the same as forgetting because the acquired fear response does not disappear unless the cue is presented in the absence of shock. B: At relatively extended intervals after extinction, conditioned fear returns. The magnitude of this "spontaneous recovery" increases with the length of the interval. C: Extinction is context specific. After acquisition in context $A$ and extinction in context $B$, extinction is expressed in context $B$, whereas conditioned fear is expressed in context A. D: Extinguished fear reappears (is "reinstated") when unsignaled presentations of the aversive event are interposed between the completion of extinction training and a subsequent test, but only if those presentations occur within the context of test. Adapted with permission from Myers and Davis. Behavioral and neural analysis of extinction. Neuron 36:567-584. Copyright (C) 2002, Cell Press. All rights reserved. ${ }^{25}$ With permission from Cell Press.

to be context specific ${ }^{11,12}$ (but see McSweeney and Swindell ${ }^{10}$ for some potentially important exceptions). Thus, it would seem that extinction is a complex phenomenon that is not well characterized as either an erasure of memory or a nonassociative response decrement.

An alternative class of theories proposes that extinction is a form of new learning that counteracts the expression of the conditioned fear response. ${ }^{13-16}$ These "inhibitory" theories suggest that fear memories are not erased in extinction but rather are inhibited in a contextdependent manner, such that the subject effectively learns that "now, in this place, the cue no longer predicts the aversive event." Hence, when the subject is tested in a context different from the one in which extinction training occurred (renewal) or when an interval of time elapses following the completion of extinction training (spontaneous recovery), the status of the cue as a predictor of the aversive event becomes ambiguous and the conditioned fear response returns. These theories emphasize retention of fear memory throughout and beyond extinction training and conceptualize extinction as new learning that suppresses fear responses.

As neuroscientists have begun to investigate extinction, they have been guided by this basic behavioral and theoretical work and have accumulated a body of data that is consistent with an inhibitory account. For example, it has been established that fear extinction, like fear acquisition, is dependent on NMDA receptors (NMDARs) and L-type voltage-gated calcium channels $(\mathrm{L}-\mathrm{VGCCs})^{17,18}$; is sensitive to modulation of second messenger systems, including kinase and phosphatase activity ${ }^{19}$; and may require protein synthesis. ${ }^{20,21}$ Moreover, some conditioned single unit responses to the fear-eliciting cue within the basolateral amygdala persist through extinction ${ }^{22}$ and can be modulated by context following extinction training in a cellular correlate of renewal. ${ }^{23}$ Extinction is postulated to reflect engagement of GABAergic interneurons and/or intercalated cell populations within the amygdala, perhaps under the control of efferent structures such as prefrontal cortex, ${ }^{24}$ so as to effectively inhibit amygdalar activation by the CS after extinction training. ${ }^{25}$

Curiously, very recent neurobiological data have emerged in support of a mechanism more consistent with an "unlearning" account of extinction, in which plasticity underlying fear memory is compromised through a process known as synaptic depotentiation. Depotentiation refers to a reversal of long-term potentiation (LTP) when low-frequency or theta-frequency stimulation is applied to afferent pathways shortly following LTP induction. Because LTP of sensory pathways onto principal neurons within the amygdala is a major candidate mechanism for the acquisition and retention of conditioned fear responses, ${ }^{26}$ processes such as depotentiation that reverse LTP would be hypothesized to erase fear memories. Lin and $\mathrm{Gean}^{27}$ have demonstrated that depotentiation occurs in the amygdala in vitro and that its induction shares some key features with fear extinction in the behaving animal. For example, both depotentiation and extinction are blocked by NMDAR and L-VGCC channel antagonists as well as inhibitors of calcineurin, a major protein phosphatase. ${ }^{27,28}$ Both depotentiation and extinction also are associated with an increase in calcineurin protein levels and enzymatic activity within the 
amygdala, as well as a reversal of fear- or LTP-associated increases in phosphorylated target molecules such as Akt. Finally, depotentiation-inducing low-frequency stimulation of the amygdala in vivo $10 \mathrm{~min}$ after fear acquisition blocks the expression of conditioned fear $24 \mathrm{~h}$ later, an effect that could be interpreted as a mimicking of extinction. ${ }^{27,28}$

These findings are impressive but puzzling because it is not clear at the present time how to reconcile them with observations of recovery of fear after extinction through spontaneous recovery, renewal, and reinstatement. Clearly, if treatments could be devised that would lead to an erasure of traumatic fear memories, this could potentially be very significant from a clinical perspective, and for this reason our laboratory currently is trying to tease apart variables that might produce an erasure of fear memory versus those that might lead to an active inhibition of fear memory in animals. For all practical purposes, however, extinction seems to be best characterized as an active inhibition of fear. Consistent with this, most pharmacological treatments that facilitate fear extinction in animals appear to do so by interacting with the major inhibitory neurotransmitter in the mammalian brain, GABA.

\section{PHARMACOLOGICAL APPROACHES TO IMPROVE EXTINCTION}

Although the study of the neurobiological basis of extinction is still in its infancy, several groups are actively pursuing pharmacological means of facilitating extinction. The general strategy is to perform basic studies in animals to determine the neurotransmitters that are critical to either the development or the expression of extinction, and then to test drugs that act on those neurotransmitters in the direction predicted to facilitate extinction. For example, if transmitter $\mathrm{X}$ is found to be critical for extinction because blocking its receptors blocks extinction, then agonists of transmitter X's receptors would be predicted to facilitate extinction, assuming that under normal conditions transmitter X's receptors are not already fully saturated. Conversely, if agonists of transmitter Y's receptors block extinction, then antagonists of transmitter Y's receptors would be predicted to facilitate extinction. Using this approach, several candidate pharmacotherapies for use as adjuncts to exposurebased CBT have been identified.

\section{The role of GABA in extinction}

As noted above, most drugs that facilitate extinction appear to do so though interactions with GABAergic neurotransmission. For this reason, a brief review of the role of GABA in extinction is warranted here.

Because extinction reflects the operation of an active inhibitory process, it is likely that GABA serves as the source of that inhibition via its actions at ionotropic $\left(\mathrm{GABA}_{\mathrm{A}}\right)$ and metabotropic $\left(\mathrm{GABA}_{\mathrm{B}}\right)$ receptors. It has been argued for some time that GABA is involved in the consolidation of fear acquisition, as GABA agonists disrupt and GABA antagonists facilitate acquisition of aversively motivated tasks. ${ }^{29}$ More recently, these findings have been extended to fear inhibition as well. For example, McGaugh et al. ${ }^{30}$ reported that postextinction training administration of the GABA antagonist picrotoxin enhanced extinction retention in a test conducted $24 \mathrm{~h}$ later, as assessed with an active avoidance paradigm. The facilitatory effect of picrotoxin was specific to animals receiving extinction training because there was no difference in test performance between nonextinguished vehicle-treated and nonextinguished drug-treated groups. In a similarly designed study, Izquierdo and Pereira ${ }^{31}$ found that pre-extinction training administration of diazepam, a benzodiazepine that acts at the GABA receptor complex to increase chloride flux, had no effect on performance within the extinction session but was associated with impaired extinction retention in a test conducted $24 \mathrm{~h}$ later.

A problem associated with the use of anxiolytic and anxiogenic compounds in studies of extinction, however, is the possibility of state dependency as opposed to a true effect on the inhibitory learning process. ${ }^{32}$ That is, it is possible that a drug administered before or immediately following extinction training produces an internal state, or drug context, that is discriminable to the animal. If testing is then conducted in the absence of this drug state, responding to an extinguished CS may be "renewed" in much the same manner as it is with a shift of physical context. Thus, suitable controls (e.g., a group of animals receiving the drug before both extinction training and the subsequent retention test) must be included in any study purporting to demonstrate a drug-dependent retardation of extinction. Indeed, it has been suggested that the apparent effects of GABAergic compounds on extinction retention actually reflect state dependency, ${ }^{33}$ although others have challenged this conclusion. ${ }^{29,34}$

In addition to its putative role in the consolidation of inhibitory learning, GABA has also been implicated in the expression of extinction. Harris and Westbrook ${ }^{35}$ demonstrated that FG-7142, an inverse agonist (i.e., a functional antagonist) of the $\mathrm{GABA}_{\mathrm{A}}$ receptor, dosedependently impaired within-session extinction and, when administered before test, blocked extinction retention in the context in which extinction training had been given but produced no effect on performance in a novel context. That is, vehicle-treated but not FG-7142-treated animals exhibited extinction retention when tested in the extinction training context, but both groups showed a similar renewal of the fear response when tested in the novel context. The lack of an increased renewal effect in FG-7142-treated animals is significant because it indi- 
A

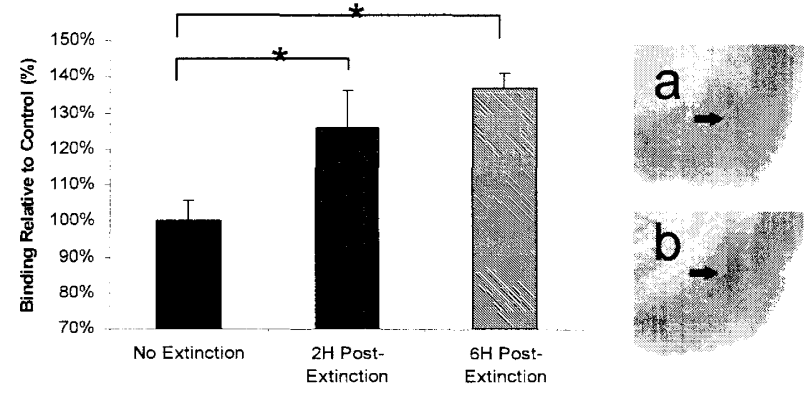

FIG. 2. Surface expression of $\mathrm{GABA}_{\mathrm{A}}$ receptors in the basolateral amygdala increases following extinction training, as indicated by increased binding of H3-Flunetrazepam. ${ }^{36} \mathrm{~A}$ : The increase in binding is evident at 2 and $6 \mathrm{~h}$ after extinction training, relative to nonextinguished controls. $\mathrm{B}$ : Comparison of autoradiographic images of brains taken from nonextinguished $(A)$ and extinguished (B) animals reveals increased binding within the baolateral amygdala. Adapted with permission from Chhatwal et al. Regulation of gephyrin and GABAA receptor binding within the amygdala after fear acquisition and extinction. $J$ Neurosci 25:502-506. Copyright (C) 2005, Society for Neuroscience. ${ }^{36}$ With permission from the Society for Neuroscience.

cates that FG-7142 did not nonspecifically increase the fear response, but rather "selectively reversed the component of extinction linked to the environmental context where extinction training had occurred" (p. 113). Separate experiments indicated that this effect could not be explained by state dependency. Thus, it appears that GABA-mediated inhibition is indeed involved in the expression of extinction, and furthermore that GABA release is itself modulated by other systems that are responsive to factors such as contextual cues.

More recently, we found that a protein called gephyrin, which contributes to the regulation of GABAergic neurotransmission by clustering $\mathrm{GABA}_{\mathrm{A}}$ receptors at the synapse, is upregulated after extinction training. ${ }^{36}$ This is in contrast to the downregulation of gephyrin after fear acquisition, which we reported previously. ${ }^{37}$ In our latest study, we replicated the acquisition finding, reporting a decrease in gephyrin protein $2 \mathrm{~h}$ after fear acquisition and a decrease in the surface expression of $\mathrm{GABA}_{\mathrm{A}}$ receptors (as evidenced by decreased binding of ${ }^{3} \mathrm{H}-$ Flunetrazepam) $6 \mathrm{~h}$ after fear acquisition in the basolateral amygdala. After extinction training, gephyrin mRNA and protein levels in the basolateral amygdala increased significantly, as did ${ }^{3} \mathrm{H}$-Flunetrazepam binding, at 2, 4, and $6 \mathrm{~h}$, respectively (FIG. 2). These results implicate gephyrin in both fear acquisition and extinction and suggest that the modulation of gephyrin and $\mathrm{GABA}_{\mathrm{A}}$ receptor surface expression in the basolateral amygdala may play a role in the experience-dependent plasticity underlying both of these types of learning. Increased levels of gephryin in the postsynaptic membrane after extinction would be expected to bring GABA receptors, normally at some distance from each other, closer together. In this way, the same amount of GABA released by the presynaptic cell would be expected to bind to a greater number of receptors, thereby enhancing GABA transmission. Conversely, after fear conditioning, the downregulation of gephyrin would be expected to result in receptors being even farther apart on the postsynaptic membrane so that the same amount of GABA release would be expected to bind to fewer receptors and hence have less of an inhibitory effect, making these postsynaptic cells more excitable. This mechanism may provide a window of increased excitability in the basolateral amygdala that may be important for consolidation of fear.

In summary, it appears that extinction is mediated to a significant extent by inhibition of the amygdala by GABA. Thus, extinction develops when sensory pathways conveying information about fear-eliciting cues come to engage GABAergic transmission within the amygdala via experience-dependent forms of synaptic plasticity (such as LTP) at synapses onto inhibitory interneurons. For this reason, inverse agonists of GABA receptors block the expression of extinction ${ }^{35}$ and gephyrin expression and $\mathrm{GABA}_{\mathrm{A}}$ receptor clustering increase within the amygdala after extinction training. ${ }^{36}$ In this light, it seems likely that the exact location of targeted manipulations of GABA receptors within the amygdala may be a significant factor in determining the direction of the effect because modulation of local GABAergic transmission that tonically inhibits the excitatory drive required for consolidation of extinction would be expected to have a different effect than modulation of GABAergic transmission in other areas that might mediate the expression of extinction. For this reason, it seems unlikely that pharmacotherapies that broadly target GABA receptors will be useful in facilitating exposure-based therapy. However, emerging evidence indicates that it may be possible to modulate more selectively either the development of plasticity onto GABAergic interneurons or the increased GABAergic transmission mediating the expression of extinction via modulation of other neurotransmitter systems. In the sections that follow, we discuss the role of glutamate, endogenous cannabinoids, dopamine, and norepinephrine in extinction, and suggest that each of these neurotransmitters may ultimately exert their effects on extinction through modulation of GABA.

\section{The role of glutamate in extinction}

Glutamate is the major excitatory neurotransmitter in the mammalian brain and acts at three major classes of receptors: AMPA, NMDA, and metabotropic glutamate receptors. ${ }^{38,39}$ Of these, NMDARs are particularly interesting because they have been implicated heavily in learning and memory in the behaving animal as well as in experience-dependent forms of plasticity such as LTP. Antagonists of NMDARs impair fear acquisition when 
administered before pairings of a cue and an aversive event. ${ }^{40}$ It is hypothesized that this is because NMDAR blockade prevents plasticity at synapses between sensory pathways conveying information about the cue to the amygdala and intrinsic amygdalar principal neurons. Because the development of extinction is believed to involve similar experience-dependent plasticity between sensory pathways and GABAergic interneurons within the amygdala, several laboratories have examined the effect of modulation of NMDAR-mediated transmission before and shortly after extinction training.

Falls et al. ${ }^{18}$ reported that intra-amygdala infusions of the NMDAR antagonist AP5 blocked extinction in a dose-dependent manner. Importantly, extinction retention was measured the following day, when AP5 had been metabolized fully and was no longer active within the brain. Other experiments indicated that this impairment could not be attributed to an effect on NMDARs outside the amygdala, to damage or destruction of the amygdala, or to an impairment of sensory transmission during extinction training. A similar blockade of extinction of contextual fear conditioning, inhibitory avoidance, and eye blink conditioning has since been reported with administration of AP5 and other NMDAR antagonists, ${ }^{19,41,42}$ and additional studies have confirmed that these effects cannot be explained by state dependency. ${ }^{43,44}$ Blockade of NMDARs after extinction training also impairs extinction, suggesting that NMDAR-mediated transmission is important for the consolidation of extinction. ${ }^{45}$

In light of these findings, the question arose as to whether it would be possible to enhance extinction by enhancing the functioning of the NMDAR. It is known that a compound called D-cycloserine (DCS) binds to the NMDAR as a partial agonist and improves its efficacy. Thus, we predicted that giving DCS before extinction training would enhance extinction. In a series of experiments conducted very similarly to those of Falls et al. ${ }^{18}$, our laboratory ${ }^{46}$ administered DCS either systemically or directly into the amygdala before extinction training and then tested retention of extinction the next day without administering any more of the drug. DCS dose-dependently enhanced extinction in rats exposed to lights in the absence of shock but not in control rats that did not receive extinction training. This indicated that the drug's facilitatory effect was specific to extinction and did not result from a general dampening of fear expression.

Ledgerwood et al. ${ }^{47}$ found that DCS given either systemically or directly into the amygdala also facilitated extinction of conditioned fear. Most interestingly, DCS could still facilitate extinction when given up to about $3 \mathrm{~h}$ after extinction training, a finding consistent with the idea that DCS facilitates consolidation of extinction. More recently, the same researchers found that DCS reduced the ability of unsignaled US presentations to disrupt extinction. ${ }^{48}$ Thus, control rats given shocks after extinction training showed the typical return of conditioned fear (reinstatement), whereas experimental rats previously treated with DCS continued to express extinction (i.e., showed much less reinstated fear).

In summary, glutamate acting at NMDARs seems to be involved critically in extinction. At first glance, this would appear to be inconsistent with the hypothesis that extinction depends primarily on GABAergic neurotransmission. However, increased GABA release from interneurons is primarily a mechanism of the expression of extinction. Glutamate is believed to be involved to the extent that sensory pathways conveying information about the extinguished cue become able to engage those interneurons through experience-dependent plasticity, i.e., as extinction develops over the course of extinction training. Consistent with this, antagonism of NMDARs has no effect on the expression of extinction that developed previously. ${ }^{43,44}$ Hence, modulation of NMDARs is hypothesized to modulate the degree to which GABAergic neurotransmission comes to be engaged in extinction, consistent with a central role for GABA in the expression of extinction.

\section{The role of cannabinoids in extinction}

Manipulation of the endogenous cannabinoid (eCB) system has become a major focus of current research, especially in the search for novel therapeutics to treat many common mental illnesses, including anxiety disorders, depression, and drug addiction. ${ }^{49,50}$ Indeed, the potential therapeutic value of cannabinoid modulation is underscored by the dense expression of the CB1 receptor in regions known to be important for anxiety and emotional learning, including the amygdala, hippocampus, and throughout the mesolimbic dopamine reward system. ${ }^{51-53}$

Recent studies of CB1 knockout mice have demonstrated that the genetic deletion of the CB1 receptor leads to increased anxiety in several well-studied measures. ${ }^{54}$ Furthermore, the elegant studies of Marsicano, Lutz, and colleagues $^{55}$ have demonstrated CB1 knockout mice also show profound deficits in extinction, whereas the acquisition of the initial fear response was normal. In the same study, the authors demonstrated that pharmacologic blockade of the $\mathrm{CB} 1$ receptor led to a similar deficit in extinction in mice, demonstrating the importance of CB1 receptor activation to extinction in mice.

Given the mounting clinical interest in modulators of the eCB system, we recently examined whether the CB1 antagonist rimonabant (SR141716A) would block extinction of fear in rats as measured with fear-potentiated startle. We then examined if administration of an agonist (WIN 55,212-2) or of an inhibitor of reuptake and breakdown (AM404) would enhance extinction of fear. In so doing, we addressed whether manipulation of the $\mathrm{eCB}$ 
system could lead to enhancements as well as decrements in extinction. ${ }^{56}$

Our results indicate that systemic administration of the CB1 antagonist rimonabant (SR141716A) before extinction training led to significant, dose-dependent decreases in extinction. Additionally, we found that, whereas the administration of the CB1 agonist WIN 55,212-2 did not appear to affect extinction, administration of AM404, an inhibitor of eCB breakdown and reuptake, led to dosedependent enhancements in extinction. In addition to showing decreased fear 1 and $24 \mathrm{~h}$ after extinction training, AM404-treated animals showed decreased shockinduced reinstatement of fear. Control experiments demonstrated the effects of AM404 could not be attributed to changes in the expression of conditioned fear, locomotion, shock reactivity, or baseline startle, as these parameters seemed unchanged by AM404. Furthermore, coadministration of rimonabant with AM404 blocked this enhancement of extinction, suggesting that AM404 was acting to increase $\mathrm{CB} 1$ receptor activation during extinction training.

Taken together, these results suggest that the CB1 receptor is a critical player in extinction learning and that the endocannabinoid system can be modulated to enhance or decrease extinction. More specifically, it seems that the endocannabinoid system may allow for a withinsession reduction of the fear response during extinction training itself, which may well be a critical step in forming a lasting extinction memory. Notably, this is consistent with the fact that postextinction administration of CB1-antagonists do not block extinction and that CB1-/- mice show impaired within session extinction, ${ }^{55}$ as well as our finding that AM404-enhanced extinction is measurable at short time points following extinction.

Because of the notion that $\mathrm{CB} 1$ receptor activation is important for the within-session reduction of conditioned fear seen during extinction training, coupled with the foregoing discussion of GABA and glutamate, we have developed a circuit diagram to serve as a working hypothesis from which to examine the interplay of these transmitter systems (FIG. 3). A key feature of this circuit diagram is that it largely separates the neural circuitry underlying fear acquisition and expression from the circuitry needed for the endocannabinoid-mediated reduction of conditioned fear, while still suggesting that both pathways receive sensory information regarding the CS and US. This separation allows for the preservation of the plasticity of the original fear memory during acquisition even after extinction learning takes place, in keeping with the wide array of evidence that extinction involves new learning, rather than erasure of the original fear memory. Additionally, this hypothetical circuit also emphasizes the importance of GABA-mediated inhibition of amygdala output neurons to the reduction in fear

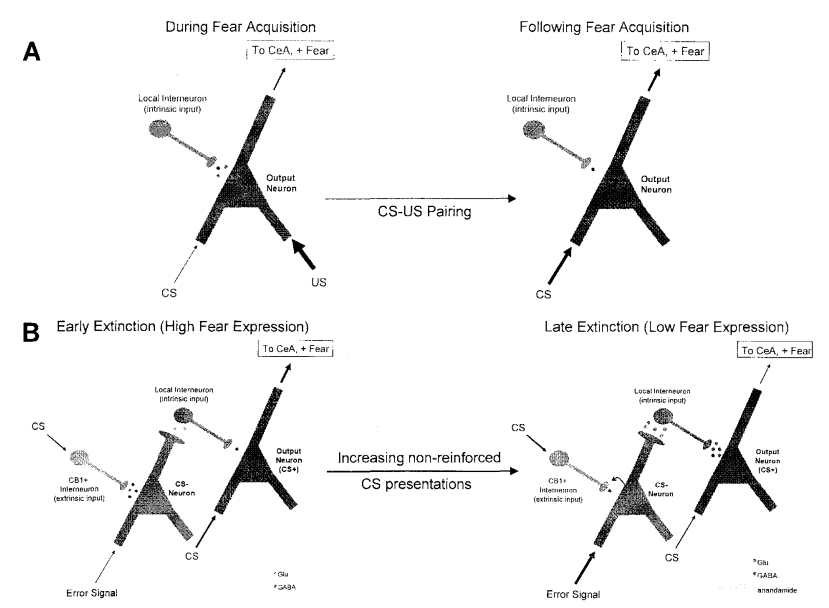

FIG. 3. A hypothetical circuit describing the neural circuitry of fear conditioning and extinction. A: In agreement with many other studies, we propose that association of the CS with the US during acquisition leads to an increase in the ability of the CS to increase the firing of amygdalar output neurons, thus allowing a post-training fear response to the CS. We further suggest that a plastic reduction in GABAergic inhibition of the output neuron enhances the ability of the CS to evoke a fear response. B: Early in extinction training (i.e., while high levels of fear to the CS are manifest), the CS is still able to efficiently drive the output neuron; later in extinction, as the behavioral error signal increases (with increasing nonreinforced CS presentations), the release of endogenous cannabinoids such as anandamide allow for the feed-forward inhibition of amygdala output neurons and a decrease in fear during the testing trial (within session extinction). Later, during the consolidation period after extinction learning, this enhancement of feed-forward inhibition becomes more stable and no longer requires further endocannabinoid release. This may occur by enhancement of several different synapses in this circuit but is ultimately dependent on increased GABAergic inhibition of the output neuron.

observed on a behavioral level. Indeed, we speculate that much of the plasticity taking place during extinction may involve modulating the feed-forward inhibition of this amygdala output neuron.

\section{The role of dopamine in extinction}

Following on a number of somewhat contradictory studies of the role in dopamine in fear acquisition, a few recent studies have emerged examining the role of dopamine in the extinction of conditioned fear. The studies of Willick and Kokkinidis ${ }^{57}$ suggest that the use of amphetamine and other enhancers of dopaminergic efflux seemed to decrease the development of extinction. Additionally, this same group has demonstrated that the D1-agonist SKF-38393 also impairs extinction; however, it has also been reported that D1-receptor knockout mice show deficits in extinction as well..$^{58}$

More recently, Nader and LeDoux ${ }^{59}$ found that the D2-agonist quinpirole both attenuated the acquisition of second-order fear conditioning, and the extinction of conditioned fear. Following up on these findings, Barad and colleagues ${ }^{60}$ endeavored to determine whether the administration of a D2-antagonist may facilitate extinction. Their results indicate that administration of 
sulpiride to mice before extinction training led to enhancements of extinction retention as compared with vehicle-treated controls. Additionally, as part of the same study, the authors were able to demonstrate that sulpiride-treated animals demonstrated extinction to widely spaced presentations of the CS, a protocol that usually provides for very low levels of extinction. ${ }^{60}$

\section{The role of norepinephrine in extinction}

The role of $\beta$-adrenergic transmission, particularly within the amygdala, as a modulator of memory consolidation has been the subject of much research. ${ }^{61,62}$ When administered systemically or directly into the amygdala, adrenergic agonists facilitate memory consolidation in Pavlovian and instrumental conditioning situations, whereas adrenergic antagonists (such as the $\beta$-adrenoreceptor antagonist propranolol) impair memory retention. This occurs when the injections are given shortly after training but not when an extended interval is interposed between the completion of training and injection, leading to the conclusion that the effect of these compounds is on consolidation of memory as opposed to encoding or some lingering drug effect on retention.

Similar effects on extinction have been reported with the administration of drugs that facilitate norepinephrine release (yohimbine) or block $\beta$-adrenergic receptors (propranolol). Cain, Blouin, and Barad ${ }^{63}$ demonstrated that extinction of contextual fear conditioning in mice was facilitated by yohimbine given immediately preextinction training, as assessed in tests conducted $24 \mathrm{~h}$ later in the absence of the drug. Yohimbine also facilitated extinction of cue conditioning (i.e., fear of a tone) when given immediately before extinction training using either a "massed" exposure protocol (5-s intervals between nonreinforced cue presentations) that produced good extinction or a "spaced" exposure protocol (20-min intervals between nonreinforced cue presentations) that did not produce extinction in the vehicle group. This did not seem to be an effect on consolidation of extinction because yohimbine did not facilitate extinction when given immediately after cue exposure. Although propranolol given before extinction training did not affect extinction retention measured $24 \mathrm{~h}$ later using the massed extinction training protocol, it impaired extinction retention $24 \mathrm{~h}$ after extinction training with the spaced extinction protocol. Under these conditions, spaced extinction trials typically lead to an increase in freezing across the session, which Cain et al. ${ }^{63}$ suggest results from an incubation of fear. Although this increase in freezing does not normally persist into the next day, they suggest that administration of propranolol during spaced extinction training allows this incubation effect to persist. More generally, Cain et al. ${ }^{63}$ suggest that cue exposure leads to both extinction and incubation of fear and that "enhancing adrenergic activity appears to tip the scales in favor of extinction, whereas impairing adrenergic activity appears to tip the scales in favor of incubation" (p. 184). Consistent with these findings, a study by Berman and Dudai ${ }^{64}$ reported that pre-extinction training infusion of propranolol directly into the insular cortex disrupted extinction of conditioned taste aversion, another form of aversive Pavlovian conditioning, and infusion of clenbuterol, a $\beta$-adrenergic agonist, reduced spontaneous recovery.

At the present time, the mechanism of noradrenergic modulation of extinction is unclear, although based on what has been learned about other modulators of extinction it seems reasonable to presume that interactions with GABAergic neurotransmission (either the development of plasticity at synapses onto interneurons or subsequent conditioned GABA release by those interneurons) are likely to be significant. Looking from a different perspective, it is interesting to speculate as to how yohimbine-induced facilitation of extinction might relate to known aspects of psychotherapy. Yohimbine is anxiogenic, and it is well known that patients need to be somewhat anxious during psychotherapy to learn to overcome their fears. In fact, benzodiazepines, which reduce fear and anxiety, may actually interfere with the benefits of psychotherapy by preventing the patient from experiencing sufficient anxiety during the therapeutic intervention.

Basic behavioral and theoretical work provide some clues as to why this might be so. Studies in rats have shown that the amount of inhibition that develops to a cue that signals the absence of an aversive footshock is directly related to the intensity of the shock or number of training trials used to condition fear. For example, in an experiment involving a paradigm called conditioned inhibition, different groups of rats learned that a $1200 \mathrm{~Hz}$ tone (A) predicted either no shock or a low or a highintensity shock, but when the tone was presented in compound with a flashing light (X), the shock would not occur (Table 1). ${ }^{5}$ Hence, the light predicted the omission of shock and is called a conditioned inhibitor or safety signal. All three groups were also trained to associate a third cue, a $250-\mathrm{Hz}$ tone (C), with a low-intensity shock. Later, the rats were tested to see how the light would affect fear of the $250-\mathrm{Hz}$ tone. Figure 4 shows a reconstruction of the results, comparing the amount of inhibition of fear to $\mathrm{C}$ when presented alone versus when compared in compound with X. Figure 4 clearly shows that the light originally paired with the high-intensity shock was much more effective in inhibiting fear to the tone than was a light originally paired with no shock or a low shock. This indicates, therefore, that to overcome high levels of fear (such as those conditioned with an intensely aversive event), it is necessary to develop high levels of inhibition. Because extinction generally appears to involve the development of inhibition, the more stress- 
TABLE 1. Experimental Design Employed by Rescorla ${ }^{5}$

\begin{tabular}{llcc}
\hline & \multicolumn{1}{c}{ Phase 1 } & Phase 2 & Test \\
\hline Group 1 & A-no shock, AX-no shock & C-0.5 mA shock & C, CX \\
Group 2 & A-0.5 mA shock, AX-no shock & C-0.5 mA shock & C, CX \\
Group 3 & A-1.0 mA shock, AX-no shock & C-0.5 mA shock & C, CX \\
\hline
\end{tabular}

ful the situation is during extinction training the greater the inhibition that should develop, provided of course that enough extinction training is given. If too little is given, then only sensitization may develop, and this could explain why short debriefing sessions given after trauma may actually not help but sometimes even worsen symptoms. ${ }^{65-67}$

But what does this have to do with yohimbine? It is known that footshock releases norepinephrine as does yohimbine. Moreover, patients with PTSD given yohimbine experience flashbacks, vivid memories of their prior trauma. ${ }^{68}$ Hence, giving yohimbine during extinction may recreate an internal state that resembles that during fear acquisition and hence vividly remind the mouse that this cue or situation was associated with a very bad event. On this basis, one would expect that the inhibition that developed would actually be stronger under these unpleasant conditions.

At first glance, therefore, it would appear that treatments that increase activation of $\beta$-adrenergic receptors might facilitate extinction in anxiety disorder patients in a clinical situation. Unfortunately, direct $\beta$-adrenergic receptor agonists do not easily cross the blood-brain barrier, so more indirect pharmacotherapies would be necessary, such as those that might increase norepinephrine cell firing or block norepinephrine reuptake. However, these would not be specific to $\beta$-adrenergic receptor

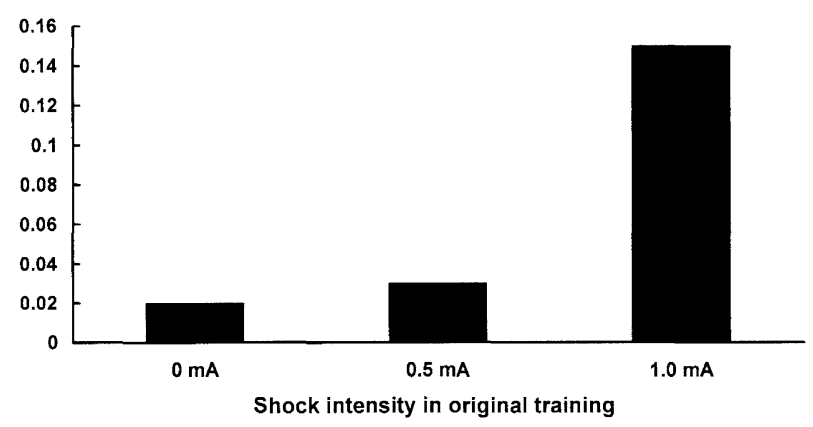

FIG. 4. Results of the experiment by Rescorla, as reported in Rescorla and Wagner (1972), ${ }^{5}$ whose design is summarized in Table 1. The bars present responding to $C$ minus responding to $\mathrm{CX}$ in test. Conditioned inhibition to $\mathrm{X}$ was greater when the $\mathrm{A}$ cue was trained with more intense shocks $(1.0 \mathrm{~mA})$, suggesting that inhibition develops more fully when the amount of fear to be inhibited is greater. Adapted with permission from Rescorla and Wagner. A theory of Pavlovian conditioning: variations in the effectiveness of reinforcement and nonreinforcement. In: Classical conditioning II (Black A, Prokasy W, eds), pp 64-99. Copyright (C) 1972, Appleton-Century-Crofts. All rights reserved. ${ }^{5}$ With permission from Robert Rescorla and Appleton-Century-Crofts. activation, and little is known about how activation of other noradrenergic receptors might affect extinction. Moreover, because yohimbine is known to cause panic attacks in patients with panic disorder ${ }^{69}$ or PTSD, ${ }^{70}$ it and other drugs in this same class would be contraindicated in such patients.

\section{CLINICAL IMPLICATIONS}

Of the drugs just discussed, only one, D-cycloserine, has yet been tested for clinical utility in facilitating exposure-based CBT in phobic patients. This primarily is because DCS already is approved by the Food and Drug Administration for use in humans for a different purpose. DCS also is the most extensively studied of these drugs in terms of its interactions with fear acquisition and extinction in animals. In addition to the findings presented above showing that DCS facilitates fear extinction, other data indicate that DCS may be particularly useful clinically because of its unique behavioral features. In this section, we will first discuss this additional animal data, then present the results of the clinical study examining the utility of DCS as a pharmacologic adjunct to exposure therapy for acrophobia (fear of heights) in humans.

\section{Does DCS cause "generalized" extinction?}

Perhaps the most surprising result from the recent studies on DCS and extinction is that DCS seems to lead to generalized extinction. ${ }^{71}$ Extinction normally is cue specific, meaning that it does not generalize to other cues that have been fear conditioned but not extinguished. In the Ledgerwood et al. study, ${ }^{71}$ rats were fear conditioned to two different cues (a light and a tone) through pairings with a very loud $(120 \mathrm{~dB})$, aversive noise. The next day, some rats were extinguished to the light and immediately thereafter were injected with DCS or saline. Other rats were injected with saline or DCS but were not given extinction training. Twenty-four hours later, all rats were tested for fear (freezing) to the light and tone. Performance to the light replicated earlier findings in that rats given DCS after extinction training exhibited less fear of the light than did either rats given saline after extinction or rats injected with DCS or saline but not extinguished. The most interesting aspect of the experiment was performance to the tone, which had not been extinguished for any of the rats. Rats given extinction training to the 
A
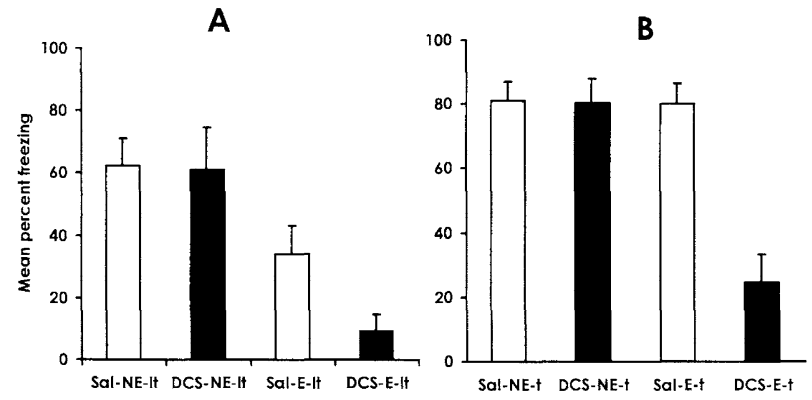

FIG. 5. Effect of DCS on extinction of conditioned freezing, as reported by Ledgerwood et al. (2005). ${ }^{71} \mathrm{~A}$ : Mean percent of time rats spent freezing to the light following either extinction training to the light or handling. B: Mean percent of time rats spent freezing to the tone following either extinction to the light or handling. Reprinted with permission from Ledgerwood et al. DCycloserine facilitates extinction of learned fear: effects on reacquisition and generalized extinction. Biol Psychiatry 57:841847. Copyright (C) 2005, Society for Biological Psychiatry. ${ }^{71}$ With permission from the Society for Biological Psychiatry.

light and injected with DCS exhibited reduced fear of the tone, i.e., generalized extinction of fear, whereas rats injected with saline after extinction training to the light or rats injected with DCS or saline but not given extinction showed no reduction in fear to the tone (FIG. 5).

One interpretation of this finding of generalized extinction after DCS administration is that DCS facilitates extinction by devaluing the US representation. To understand this concept fully, some background information is required. It has been suggested that Pavlovian conditioning occurs when an organism forms mental or neural representations of the stimuli involved (the fear-inducing cue, also called a conditioned stimulus or CS, and the aversive event, also called an unconditioned stimulus or US) and then creates associations (bonds) between them. When the CS-US association is formed, physical presentation of the CS activates the CS representation directly and the US representation indirectly via the associative bond between CS and US representations. The US representation, when activated associatively, then triggers conditioned fear responses (CRs).

Association formation traditionally has been viewed as the primary mechanism of Pavlovian conditioning, although modulation of the CS and US representations also may play a role. Devaluation of the US representation, meaning a reduction in the strength or salience of the mental or neural representation of the aversive event, was suggested by Rescorla and Heth ${ }^{9}$ to be particularly important in extinction. Among the evidence they presented in support of this notion is an experiment by Rescorla $^{72}$ in which rats received pairings of a cue (CS) with a loud noise (US). Afterward, some rats were habituated to the loud noise, i.e., they were presented with the loud noise repeatedly in the absence of the cue. Those rats that were habituated to the noise subsequently exhibited less fear of the cue (measured by lick suppres- sion) than those rats not habituated to the noise, suggesting that the representation of the loud noise had been weakened through repeated exposures to that stimulus. It should be noted that the drop in fear to the cue occurred despite the fact that no further pairings of the cue and the loud noise occurred, and no extinction training to the cue was given between fear conditioning and test. Rescorla and $\mathrm{Heth}^{9}$ suggested that extinction after nonreinforced presentations of the cue might result from a similar process of US devaluation. That is, if it is assumed that the cue associatively activates the mental or neural representation of the loud noise during the extinction trials, then the absence of physical noise presentations might result in a devaluation of the representation of the noise. As a result, subsequent presentations of the cue (as in a test conducted after extinction training) produce little or no fear because the cue activates a representation of the noise that is too weak to trigger fear responses.

Returning to the observation of generalized extinction after administration of DCS, Lederwood et al. ${ }^{71}$ proposed that US devaluation might account for that phenomenon if it is assumed (as most theories of Pavlovian conditioning do) that separate representations of the light and tone cues each form an associative bond with a single representation of the US. Devaluation of the US representation through extinction of the light would then "transfer" to the tone because physical presentation of the tone after extinction to the light will associatively activate a weakened US representation that is less able to elicit a conditioned fear response. To examine this hypothesis further, in a separate experiment designed similarly to that of Rescorla ${ }^{72}$ reported above, Ledgerwood et $\mathrm{al}^{71}$ trained rats to fear a light and a tone through pairings with a loud noise US. Some rats were then habituated to the loud noise. In a subsequent test, the rats habituated to the loud noise exhibited much less freezing to the light and tone than did rats that were not habituated. In other words, the rats habituated to the noise before test exhibited a pattern of performance (generalized dampening of fear) just like that seen in rats given DCS after extinction training with a visual cue but then tested later with both a visual and auditory cue (generalized extinction).

The US devaluation hypothesis of the modulation of extinction by DCS is attractive but not without its problems. For example, it does not easily explain why DCS also prevented reinstatement because unsignaled presentations of a US following extinction would be expected to revive the degraded representation of the shock, and hence lead to a reinstatement of the fear response to the cue previously paired with that shock. ${ }^{9}$ On the other hand, reinstatement is context specific (i.e., unsignaled shock presentations must be given in the same context in which rats ultimately are tested, otherwise they are ineffective), which would seem to suggest that US devalua- 
tion is not the only mechanism of or even a significant contributor to reinstatement. ${ }^{8}$ Another possibility suggested by Richardson et al. ${ }^{73}$ is that DCS-injected rats develop an extremely strong (and possibly context-independent) inhibitory CS-US association. For example, Denniston et al. ${ }^{74}$ reported that massive extinction (800 extinction trials) reduced renewal. If DCS causes extinction to become context independent after only a few trials, then reinstatement might be more difficult to demonstrate in these rats compared with saline-treated rats. That is, it may be necessary to provide stronger, or more, shock exposures to observe reinstatement in the DCStreated animals. Clearly, further research is required to examine this possibility.

In clinical terms, generalized extinction after DCS may be beneficial in the sense that extinction of a single cue might generalize to other cues that acquired fear at the same time, which could greatly facilitate therapy for complex anxiety disorders such as PTSD in which many cues trigger fear reactions. For example, a person who witnessed the World Trade Center disaster and developed PTSD might have multiple fear triggers including odors, sounds, and certain visual cues, all of which were in effect conditioned in a single "trial" and might be particularly amenable to generalized extinction via extinction of just one cue. Much more research is required, however, to examine carefully the situations in which generalized extinction might be beneficial versus those in which it could actually be detrimental. It seems clear, for example, that generalized extinction to cues that trigger adaptive fear responses would not be wise.

\section{DCS does not seem to facilitate fear conditioning and may even reduce it}

If DCS is so effective in facilitating learning, then one might wonder whether it could actually be harmful if combined with exposure-based psychotherapy. For example, bringing to mind awful memories of a traumatic event can lead to sensitization rather than extinction if a full therapeutic exposure is not carried out. ${ }^{65-67}$ Perhaps sensitization would be exacerbated by DCS by strengthening the fearful memories. Thus far, none of us has seen any evidence of this in our rodent studies, nor was any evidence of this observed in our clinical study (see below). In fact, we found that DCS does not facilitate fear conditioning under the conditions we use (Walker, D. L., and M. Davis, unpublished observations).

Why might DCS not facilitate fear acquisition? DCS is an analog of a naturally occurring chemical in the brain, D-serine. D-Serine and glycine bind to the same site on the NMDAR, as does DCS, and make the receptor work better. So it is possible that NMDARs involved in fear conditioning are already saturated with D-serine or glycine, making these receptors work optimally. This optimal functioning would be adaptive because it is very important for all animals to learn quickly what stimuli are dangerous so as to avoid them in the future. If the receptors are already saturated, DCS would not be able to have any further effect. In fact, DCS is actually less effective than either D-serine or glycine and so, if the site on the NMDARs involved in fear conditioning is fully saturated, DCS might actually reduce the activity of the receptors by displacing the more effective endogenous chemicals. This could explain why DCS appears to actually inhibit fear conditioning in some situations.

But how does this explain the ability of DCS to facilitate extinction? Perhaps the NMDARs involved in extinction are different from those involved in fear conditioning (e.g., on different neurons), and perhaps those involved in extinction are not saturated with glycine or D-serine. This would suggest these particular NMDARs do not work as efficiently, an explanation for why extinction takes much longer to develop than fear conditioning. But because these receptors are not already saturated, then the effect of giving DCS would be to facilitate NMDA transmission and, therefore, extinction.

In conclusion, with clinical use of DCS there does not seem to be any danger of inadvertently facilitating fear rather than extinction because there is no evidence of an acquisition effect in animals.

\section{A clinical test of combining DCS with behavioral exposure therapy for acrophobia}

Recently, we tested whether DCS given in combination with exposure therapy for the treatment of specific phobia in humans would improve the effectiveness of this therapy. ${ }^{75} \mathrm{We}$ wished to examine the ability of DCS to enhance exposure therapy in humans using the most optimally controlled form of psychotherapeutic learning available. Virtual reality exposure (VRE) therapy is ideal for clinical research assessment because exposure and testing is identical between patients, is well controlled by the therapist, and occurs within the spatial and temporal confines of the limited therapy environment. ${ }^{76}$ This method has proven to be successful for the treatment of specific phobias as well as PTSD. ${ }^{76-79}$ With VRE for fear of heights, we used a virtual glass elevator in which participants stood while wearing a VRE helmet and were able to peer over a virtual railing. Previous work has shown improvements on all acrophobia outcome measures for treated as compared with untreated groups after seven weekly therapy sessions. ${ }^{76}$ To examine whether DCS would enhance the learning that occurs during exposure therapy for humans with specific phobia, we enrolled 28 volunteer participants who were diagnosed with acrophobia by DSM-IV. ${ }^{75}$ Participants were randomly assigned to three treatment groups, placebo + VRE therapy, or DCS + VRE therapy at two different doses of DCS (50 or $500 \mathrm{mg}$ ). Treatment condition was double blinded, such that the subjects, therapists, and 
C
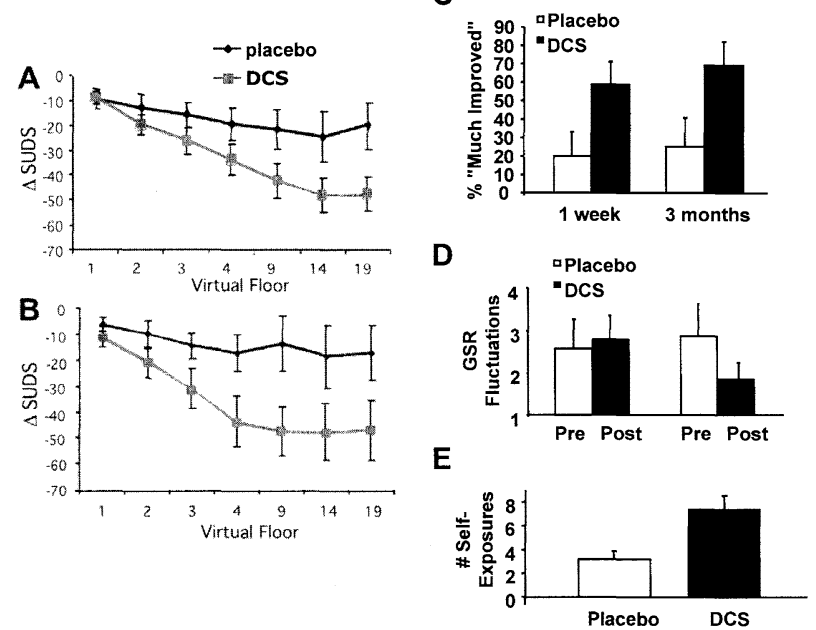

FIG. 6. Results of the clinical study with D-cycloserine as an adjunct to exposure therapy for the treatment of acrophobia. ${ }^{75}$ $A$ : Change in subjective units of discomfort (SUDS) from pre- to post-test after two therapy sessions that occurred approximately 1 week before this short-term follow-up assessment. Decrease in SUDS level is shown for each floor (1-19) of the virtual glass elevator. B: Change in SUDS from pre to post-test at the 3-month long-term follow-up assessment. C: Percent of patients in the DCS or placebo groups reporting "Much improved" at 3-month follow-up. D: Self-report of number of in vivo exposures to heights since the treatment 3 months earlier. $\mathrm{E}$ : Number of spontaneous galvanic skin fluctuations in the simulated glass elevator before and 1-week after therapy in the two groups. Adapted with permission from Ressler et al. Cognitive enhancers as adjuncts to psychotherapy: use of D-cycloserine in phobic individuals to facilitate extinction of fear. Arch Gen Psychiatry 61:1136-1144. Copyright (C) 2004, American Medical Association. All rights reserved. ${ }^{75}$ With permission from AMA.

assessors were not aware of assigned study medication condition. Although we used two different doses of DCS, preliminary analysis of our data indicated that there were no significant differences between the 50- and 500-mg drug groups for the primary outcome measures of acrophobia. Therefore, we combined the two drug groups for analysis.

Participants underwent two therapy sessions, which is a suboptimal amount of exposure therapy for acrophobia. $^{76}$ They were instructed to take a single pill of study medication 2-4 $\mathrm{h}$ before each therapy session, such that only two pills were taken for the entire study. A posttreatment assessment was performed within a week after the two therapy sessions, and an additional follow-up assessment was performed 3 months after the therapy.

At both 1-2 weeks and 3 months after treatment, subjects who received DCS in conjunction with VRE therapy had significantly enhanced decreases in fear within the virtual environment (FIG. 6, A and B; $P<0.05$ ). Patients who received DCS in conjunction with therapy felt that they had improved significantly more than the placebo group in their overall acrophobia symptoms at the 3-month follow-up (FIG. 6C). Furthermore, within the virtual environment, skin conductance fluctuations, a psychophysiological measure of anxiety, was significantly decreased in the group that received DCS in conjunction with therapy (FIG. 6D).

One of the cardinal features of extinction in animal models is the context specificity of the extinction environment. However, Richardson and colleagues ${ }^{71}$ have demonstrated that DCS enhancement of extinction in rats appears to lead to generalization across cues. We therefore wondered if the decreased fear of heights found within the virtual environment would generalize to other settings. This question was assessed in two ways, first by asking questions related to the subject's fear of heights in the real world, and secondly by assessing how much the subjects had decreased their avoidance of heights since the treatment. We found that patients' self-exposure to heights in the "real world" had increased, suggesting decreased avoidance (FIG. 6E).

Our data indicate that participants receiving DCS experienced no increase in anxiety or fear during the exposure paradigm so that the enhancement of extinction is not due simply to enhanced intensity of exposure. Participants in the DCS group showed some evidence of enhanced extinction after only a single dose of medication and therapy. After two doses of medication and therapy, they showed significant reductions in levels of fear to the specific exposure environment. Finally, we found that 3 months after the two treatment sessions, the DCS participants showed significant improvements on all acrophobia outcome measures, their own self-exposures in the real world, and their impression of clinical self-improvement relative to participants who received placebo. ${ }^{75}$

It is important to note that the timing of dosing of DCS may be critical in the use of this agent in the augmentation of exposure therapy. Despite animal studies suggesting enhancement of spatial learning, ${ }^{80-83}$ the studies of human trials in patients with dementia have found only minor improvements ${ }^{84,85}$ or no significant effect on memory enhancement. ${ }^{86-88}$ We believe that a principal difference between those studies, our human acrophobia study, and the animal literature is the frequency and chronicity of drug dosing. The human memory enhancement studies used daily dosing for weeks to months compared with single dosing before the learning event in animal experiments and in our exposure study. In fact, Quartermain and colleagues ${ }^{81}$ explicitly examined single versus chronic dosing of DCS in animals for improvement of learning. They found that a single dose of drug before training enhanced the learning of the task, whereas 15 days of drug before the task had essentially no effect on the learning. This has very recently been explicitly tested with extinction by Richardson and colleagues $^{89}$ in which they found that rats receiving five doses of DCS on an every other day schedule received no benefit when given DCS in combination with an extinc- 
tion training session compared with significant facilitation of extinction with acute dosing.

Interestingly, it is now accepted that most psychiatric medications have their intended psychotropic effect not through their acute mechanisms, but through chronic mechanisms that often involve receptor, cellular, and systemic regulatory mechanisms that are quite distinct from the acute pharmacologic drug effect. However, in the case of DCS augmentation of exposure therapy, chronic treatment may actually result in a loss of efficacy due to tachyphylaxis, as well as other regulatory phenomena that are likely to occur with prolonged activation of the NMDAR. Thus, in contrast to other psychotropic medication, to achieve the intended effect of enhancing NMDAR activity, DCS may need to be taken on an acute schedule specifically in combination with the exposurebased treatment.

\section{CONCLUSION}

In conclusion, we have applied some of the lessons learned in extinction training in animals to humans with exciting results. The use of a specific pharmacological intervention to enhance the beneficial effects of psychotherapy represents a new paradigm in psychiatry. Although we have focused on the effects of a partial NMDA antagonist in the present review, it should be noted that other agents can enhance extinction of learned fear in nonhuman animals. Future clinical studies on potential pharmacological enhancement of exposurebased therapy should focus on agents that enhance the learning that occurs during the exposure sessions, rather than on agents that reduce the anxiety experienced during those sessions.

Acknowledgments: This work was supported by National Institute of Mental Health Grants MH-047840 (to M.D.), MH069884 (to K.J.R.), and MH070218 (to J.P.C.); a National Science Foundation Grant, IBN-987675 for the Science and Technology Center Program, Center for Behavioral Neuroscience; and The Yerkes National Primate Center P-51 Base Grant. Drs. Davis and Ressler have submitted a patent for the use of D-cyloserine for the specific enhancement of learning during psychotherapy and have founded a company called SyneurRx to develop this technology. Dr. Davis and Dr. Ressler are entitled to royalty derived from SyneurRx's sale of products related to the research described in this paper. The terms of this arrangement have been reviewed and approved by Emory University in accordance with its conflict of interest policies.

\section{REFERENCES}

1. Rodrigues SM, Schafe GE, LeDoux JE. Molecular mechanisms underlying emotional learning and memory in the lateral amygdala. Neuron 44:75-91, 2004.

2. Aggleton JP. The amygdala. New York: Oxford University Press, 2000.

3. Herman JL. Trauma and recovery. New York: BasicBooks, 1992.

4. Gale GD, Anagnostaras SG, Godsil BP, Mitchell S, et al. Role of the basolateral amygdala in the storage of fear memories across the adult lifetime of rats. $J$ Neurosci 24:3810-3815, 2004.

5. Rescorla RA, Wagner AR. A theory of Pavlovian conditioning: variations in the effectiveness of reinforcement and nonreinforcement. In: Classical conditioning II (Black A, Prokasy W, eds), pp 64-99. New York: Appleton-Century-Crofts, 1972.

6. Hawkins RD, Kandel ER. Is there a cell-biological alphabet for simple forms of learning? Psychol Rev 91:375-391, 1984.

7. Robbins S. Mechanisms underlying spontaneous recovery in autoshaping. J Exp Psychol Anim Behav Process 16:235-249, 1990.

8. Bouton ME, Bolles RC. Role of conditioned contextual stimuli in reinstatement of extinguished fear. J Exp Psychol Anim Behav Process 5:368-378, 1979.

9. Rescorla RA, Heth CD. Reinstatement of fear to an extinguished conditioned stimulus. J Exp Psychol Anim Behav Process 1:88-96, 1975.

10. McSweeney FK, Swindell S. Common processes may contribute to extinction and habituation. J Gen Psychol 129:364-400, 2002.

11. Davis M, File SE. Intrinsic and extrinsic habituation and sensitization: implications for the design and interpretation of experiments. In: Habituation and the behaving organism (Peeke H, Petrinovich L, eds), pp 287-323. New York: Academic Press, 1984.

12. Marlin NA, Miller RR. Associations to contextual stimuli as a determinant of long-term habituation. J Exp Psychol Anim Behav Process 7:313-333, 1981.

13. Bouton ME. Context, time, and memory retrieval in the interference paradigms of Pavlovian learning. Psychol Bull 114:80-99, 1993.

14. Konorski J. Conditioned reflexes and neuronal organization. London: Cambridge University Press, 1948.

15. Pavlov I. Conditioned reflexes. Oxford: Oxford University Press, 1927.

16. Wagner AR. SOP: a model of automatic memory processing in animal behavior. In: Information processing in animals: memory mechanisms (Spear N, ed). Hillside, NJ: Lawrence Erlbaum Associates, 1981.

17. Cain CK, Blouin AM, Barad M. L-type voltage-gated calcium channels are required for extinction, but not for acquisition or expression, of conditional fear in mice. J Neurosci 22:9113-9121, 2002.

18. Falls WA, Miserendino MJ, Davis M. Extinction of fear potentiated startle: blockade by infusion of an NMDA antagonist into the amygdala. J Neurosci 12:854-863, 1992.

19. Szapiro G, Vianna MR, McGaugh JL, Medina JH, et al. The role of NMDA glutamate receptors, PKA, MAPK and CaMKII in the hippocampus in extinction of conditioned fear. Hippocampus 13: 53-58, 2003.

20. Santini E, Ge H, Ren K, Pena de Ortiz S, et al. Consolidation of fear extinction requires protein synthesis in the medial prefrontal cortex. J Neurosci 24:5704-5710, 2004.

21. Lattal KM, Abel T. Different requirements for protein synthesis in acquisition and extinction of spatial preferences and contextevoked fear. J Neurosci 21:5773-5880, 2001.

22. Repa JC, Muller J, Apergis J, Desrochers TM, et al. Two different lateral amygdala cell populations contribute to the initiation and storage of memory. Nat Neurosci 4:724-731, 2001.

23. Hobin JA, Goosens KA, Maren S. Context-dependent neuronal activity in the lateral amygdala represents fear memories after extinction. J Neurosci 23:8410-8416, 2003.

24. Quirk GJ, Russo GK, Barron JL, Lebron K. The role of ventromedial prefrontal cortex in the recovery of extinguished fear. J Neurosci 20:6225-6231, 2000.

25. Myers KM, Davis M. Behavioral and neural analysis of extinction. Neuron 36:567-584, 2002.

26. Schafe GE, Nader K, Blair HT, LeDoux JE. Memory consolidation of Pavlovian fear conditioning: a cellular and molecular perspective. Trends Neurosci 24:540-546, 2001.

27. Lin $\mathrm{CH}$, Lee CC, Gean PW. Involvement of a calcineurin cascade in amygdala depotentiation and quenching of fear memory. Mol Pharmacol 63:44-52, 2003.

28. Lin $\mathrm{CH}$, Yeh $\mathrm{SH}$, Leu TH, Chang WC, et al. Identification of calcineurin as a key signal in the extinction of fear memory. J Neurosci 23:1574-1579, 2003. 
29. Castellano C, McGaugh JL. Retention enhancement with posttraining picrotoxin: lack of state dependency. Behav Neural Biol 51: 165-170, 1989

30. McGaugh JL, Introini-Collison IB, Nagahara AH, Cahill L, et al. Involvement of the amygdaloid complex in neuromodulatory influences on memory storage. Neurosci Biobehav Rev 14:425-431, 1990.

31. Izquierdo I, Pereira ME. Post-training memory facilitation blocks extinction but not retroactive interference. Behav Neural Biol 51: 108-113, 1989.

32. Overton D. Experimental methods for the study of state-dependent learning. Fed Proc 33:1800-1813, 1974.

33. Bouton ME, Kenney FA, Rosengard C. State-dependent fear extinction with two benzodiazepine tranquilizers. Behav Neurosci 104:44-55, 1990.

34. Castellano C, McGaugh JL. Effects of post-training bicuculline and muscimol on retention: lack of state dependency. Behav Neural Biol 54:156-164, 1990.

35. Harris JA, Westbrook RF. Evidence that GABA transmission mediates context-specific extinction of learned fear. Psychopharmacology (Berl) 140: 105-115, 1998.

36. Chhatwal JP, Myers KM, Ressler KJ, Davis M. Regulation of gephyrin and GABAA receptor binding within the amygdala after fear acquisition and extinction. J Neurosci 25:502-506, 2005.

37. Ressler KJ, Paschall G, Zhou XL, Davis M. Regulation of synaptic plasticity genes during consolidation of fear conditioning. $\mathrm{J} \mathrm{Neu}$ rosci 22:7892-902, 2002.

38. Carlson PJ, Singh JB, Zarate CA Jr, Drevets WC, Manji HK. Neural circuitry and neuroplasticity in mood disorders: insights for novel therapeutic targets. NeuroRx 3:22-41, 2006.

39. Pittenger C, Krystal JH, Coric V. Glutamate-modulating drugs as novel pharmacotherapeutic agents in the treatment of obsessivecompulsive disorder. NeuroRx 3:69-81, 2006.

40. Miserendino MJD, Sananes CB, Melia KR, Davis M. Blocking of acquisition but not expression of conditioned fear-potentiated startle by NMDA antagonists in the amygdala. Nature 345:716-718, 1990.

41. Kehoe E, Macrae M, Hutchinson C. MK-801 protects conditioned response from extinction in the rabbit nictitating membrane preparation. Psychobiology 24:127-135, 1996.

42. Lee H, Kim J. Amygdalar NMDA receptors are critical for new fear learning in previously fear-conditioned rats. J Neurosci 18 : 8444-8454, 1998.

43. Baker JD, Azorlosa JL. The NMDA antagonist MK-801 blocks the extinction of Pavlovian fear conditioning. Behav Neurosci 110: 618-620, 1996.

44. Cox J, Westbrook R. The NMDA receptor antagonist MK-801 blocks acquisition and extinction of conditioned hypoalgesia responses in the rat. $Q J$ Exp Psychol 47B:187-210, 1994.

45. Santini E, Muller RU, Quirk GJ. Consolidation of extinction learning involves transfer from NMDA-independent to NMDA-dependent memory. J Neurosci 21:9009-9017, 2001.

46. Walker DL, Ressler KJ, Lu KT, Davis M. Facilitation of conditioned fear extinction by systemic administration or intra-amygdala infusions of D-cycloserine as assessed with fear-potentiated startle in rats. J Neurosci 22:2343-2351, 2002.

47. Ledgerwood L, Richardson R, Cranney J. Effects of D-cycloserine on extinction of conditioned freezing. Behav Neurosci 117:341349, 2003.

48. Ledgerwood L, Richardson R, Cranney J. D-Cycloserine and the facilitation of extinction of conditioned fear: consequences for reinstatement. Behav Neurosci 118:505-513, 2004.

49. Porter AC, Felder CC. The endocannabinoid nervous system: unique opportunities for therapeutic intervention. Pharmacol Ther 90:45-60, 2001.

50. Kathuria S, Gaetani S, Fegley D, Valino F, et al. Modulation of anxiety through blockade of anandamide hydrolysis. Nat Med 9:76-81, 2003.

51. Katona I, Sperlagh B, Sik A, Kafalvi A, et al. Presynaptically located CB1 cannabinoid receptors regulate GABA release from axon terminals of specific hippocampal interneurons. J Neurosci 19:4544-4558, 1999.

52. Freund TF, Hajos N. Excitement reduces inhibition via endocannabinoids. Neuron 38:362-365, 2003.
53. Simon AB, Gorman JM. Advances in the treatment of anxiety: targeting glutamate. NeuroRx 3:57-68, 2006.

54. Haller J, Bakos N, Szirmay M, Ledent C, et al. The effects of genetic and pharmacological blockade of the CB1 cannabinoid receptor on anxiety. Eur J Neurosci 16:1395-1398, 2002.

55. Marsicano G, Wotjak CT, Azad SC, Bisogno T, et al. The endogenous cannabinoid system controls extinction of aversive memories. Nature 418:530-534, 2002.

56. Chhatwal JP, Davis M, Maguschak KA, Ressler KJ. Enhancing cannabinoid neurotransmission augments the extinction of conditioned fear. Neuropsychopharmacology 30:516-524, 2005.

57. Willick ML, Kokkinidis L. Cocaine enhances the expression of fear potentiated startle: evaluation of state-dependent extinction and the shock sensitization of acoustic startle. Behav Neurosci 109:929-938, 1995.

58. El-Ghundi M, O'Dowd BF, George SR. Prolonged fear responses in mice lacking dopamine D1 receptor. Brain Res 892:86-93, 2001.

59. Nader K, LeDoux JE. Inhibition of the mesoamygdala dopaminergic pathway impairs the retrieval of conditioned fear associations. Behav Neurosci 113:891-901, 1999.

60. Ponnusamy R, Nissim HA, Barad M. Systemic blockade of D2like dopamine receptors facilitates extinction of conditioned fear in mice. Learn Mem 12:399-406, 2005.

61. McGaugh JL, Cahill, L, Roozendaal B. Involvement of the amygdala in memory storage: interaction with other brain systems. Proc Natl Acad Sci USA 93:13508-13514, 1996.

62. McGaugh JL. Memory-a century of consolidation. Science 287: 248-251, 2000.

63. Cain CK, Blouin AM, Barad M. Adrenergic transmission facilitates extinction of conditional fear in mice. Learn Mem 11:179187, 2004.

64. Berman DE, Dudai Y. Memory extinction, learning anew, and learning the new: dissociations in the molecular machinery of learning in cortex. Science 291:2417-2419, 2001.

65. Bisson JI. Post-traumatic stress counselling. Br J Hosp Med 57: 112, 1997.

66. Mayou RA, Ehlers A, Hobbs M. Psychological debriefing for road traffic accident victims. Three-year follow-up of a randomised controlled trial. Br J Psychiatry 176:589-593, 2000.

67. Rose S, Bisson JI, Churchill R, Wessely S. Psychological debriefing for preventing posttraumatic stress disorder (PTSD). Cochrane Database Syst Rev 2:CD000560, 2001.

68. Southwick SM, Morgan A, Nagy LM, Bremner D, et al. Trauma related symptoms in veterans of Operation Desert Storm: a preliminary report. Am J Psychiatry 150:1524-1528, 1993.

69. Charney DS, Woods SW, Goodman WK, Heninger GR. Neurobiological mechanisms of panic anxiety: biochemical and behavioral correlates of yohimbine-induced panic attacks. Am J Psychiatry 144:1030-1036, 1987.

70. Southwick SM, Krystal JH, Morgan CA, Johnson D, et al. Abnormal noradrenergic function in posttraumatic stress disorder. Arch Gen Psychiatry 50:266-274, 1993.

71. Ledgerwood L, Richardson R, Cranney J. D-Cycloserine facilitates extinction of learned fear: effects on reacquisition and generalized extinction. Biol Psychiatry 57:841-847, 2005.

72. Rescorla RA. Effect of US habituation following conditioning. J Comp Physiol Psychol 82:137-143, 1973.

73. Richardson R, Ledgerwood L, Cranney J. Facilitation of fear extinction by D-cycloserine: theoretical and clinical implications. Learn Mem 11:510-516, 2004.

74. Denniston J, Chang R, Miller R. Massive extinction treatment attenuates the renewal effect. Learn Motiv 34:68-86, 2003.

75. Ressler KJ, Rothbaum BO, Tannenbaum L, Anderson P, et al. Cognitive enhancers as adjuncts to psychotherapy: use of D-cycloserine in phobic individuals to facilitate extinction of fear. Arch Gen Psychiatry 61:1136-1144, 2004.

76. Rothbaum BO, Hodges LF, Kooper R, Opdyke D, et al. Effectiveness of computer-generated (virtual reality) graded exposure in the treatment of acrophobia. Am J Psychiatry 152:626-628, 1995.

77. Rothbaum BO, Hodges LF, Ready D, Graap K, et al. Virtual reality exposure therapy for Vietnam veterans with posttraumatic stress disorder. J Clin Psychiatry 62:617-622, 2001. 
78. Rothbaum BO, Schwartz AC. Exposure therapy for posttraumatic stress disorder. Am J Psychother 56:59-75, 2002.

79. Rothbaum BO, Hodges L, Kooper R. Virtual reality exposure therapy. J Psychother Pract Res 6:219-226, 1997.

80. Baxter M, Lanthorn T, Frick K, Golski S, et al. D-Cycloserine, a novel cognitive enhancer, improves spatial memory in aged rats. Neurobiol Aging 15:207-213, 1994.

81. Quartermain D, Mower J, Rafferty M, Herting R, et al. Acute but not chronic activation of the NMDA-coupled glycine receptor with D-cycloserine facilitates learning and retention. Eur J Pharmacol 257:7-12, 1994.

82. Schuster GM, Schmidt WJ. D-Cycloserine reverses the working memory impairment of hippocampal-lesioned rats in a spatial learning task. Eur J Pharmacol 224:97-98, 1992.

83. Thompson LT, Moskal JR, Disterhoft JF. Hippocampus dependent learning facilitated by a monoclonal antibody or D-cycloserine. Nature 359:638-641, 1992.
84. Schwartz BL, Hashtroudi S, Herting RL, Schwartz P, et al D-Cycloserine enhances implicit memory in Alzheimer patients. Neurology 46:420-424, 1996.

85. Tsai G, Falk W, Gunther J. A preliminary study of D-cycloserine treatment in Alzheimer's disease. J Neuropsychiatry Clin Neurosci 10:224-226, 1998.

86. Laake K, Oeksengaard AR. D-Cycloserine for Alzheimer's disease. Cochrane Database Syst Rev 2:CD003153, 2002.

87. Randolph C, Roberts J, Tierney M, Bravi D, et al. D-Cycloserine treatment of Alzheimer's disease. Alzheimers Dis Assoc Disord 8:198-205, 1994.

88. Fakouhi T, Jhee S, Sramek J, Benes C, et al. Evaluation of cycloserine in the treatment of Alzheimer's disease. J Geriatric Psych Neurol 8:226-230, 1995.

89. Parnas AS, Weber M, Richardson R. Effects of multiple exposures to D-cycloserine on extinction of conditioned fear in rats. Neurobiol Learn Mem 83:224-231, 2005. 